\title{
Early delirium after cardiac surgery: an analysis of incidence and risk factors in elderly ( $\geq 65$ years) and very elderly ( $\geq 80$ years) patients
}

\author{
Katarzyna Kotfis' \\ Aleksandra Szylińska ${ }^{2}$ \\ Mariusz Listewnik ${ }^{3}$ \\ Marta Strzelbicka' \\ Mirosław Brykczyński \\ Iwona Rotter ${ }^{2}$ \\ Maciej Żukowski' \\ 'Department of Anesthesiology, \\ Intensive Therapy and Acute \\ Intoxications, Pomeranian Medical \\ University, Szczecin, Poland; \\ ${ }^{2}$ Department of Medical Rehabilitation \\ and Clinical Physiotherapy, \\ Pomeranian Medical University, \\ Szczecin, Poland; ${ }^{3}$ Department of \\ Cardiac Surgery, Pomeranian Medical \\ University, Szczecin, Poland
}

This article was published in the following Dove Press journal: Clinical Interventions in Aging

\begin{abstract}
Introduction: Postoperative delirium is a common complication of cardiac surgery associated with increased mortality, morbidity, and long-term cognitive dysfunction. The aim of this study was to identify incidence and risk factors of delirium in elderly ( $\geq 65$ years) and very elderly ( $\geq 80$ years) patients undergoing major cardiac surgery.
\end{abstract}

Materials and methods: We performed a retrospective cohort analysis of prospectively collected data from a register of the cardiac surgery department of a tertiary referral university hospital between 2014 and 2016. Analysis was performed in two groups, $\geq 65$ years and $\geq 80$ years.

Results: We analyzed 1,797 patients $\geq 65$ years, including 230 (7.24\%) patients $\geq 80$ years. Delirium was diagnosed in $21.4 \%(384 / 1,797)$ of patients above 65 years, and in $33.5 \%(77 / 230)$ of octogenarians. Early mortality did not differ between patients with and without delirium. Intensive care unit (ICU) stay ( $p<0.001)$, hospital stay $(p<0.001)$, and intubation time $(p=0.002)$ were significantly longer in patients undergoing cardiac surgery $\geq 65$ years with delirium. According to multivariable analysis, $\geq 65$ years, age (odds ratio [OR] 1.036, $p=0.002$ ), low ejection fraction (OR 1.634, $p=0.035)$, diabetes $(1.346, p=0.019)$, and extracardiac arteriopathy (OR 1.564, $p=0.007$ ) were found to be independent predictors of post-cardiac surgery delirium. Postoperative risk factors for developing delirium $\geq 65$ years were atrial fibrillation (1.563, $p=0.001$ ), postoperative pneumonia (OR 1.896, $p=0.022$ ), elevated postoperative creatinine (OR 1.384, $p=0.004$ ), and prolonged hospitalization (OR 1.019, $p=0.009$ ).

Conclusion: Patients above 65 years of age with postoperative delirium have poorer outcome and are more likely to have prolonged hospitalization and ICU stay, and longer intubation times, but 30-day mortality is not increased. In our study, eight independent risk factors for development of post-cardiac surgery delirium were age, low ejection fraction, diabetes, extracardiac arteriopathy, postoperative atrial fibrillation, pneumonia, elevated creatinine, and prolonged hospitalization time.

Keywords: elderly, age, delirium, cardiac surgery, risk factors, mortality, outcome

\section{Introduction}

Postoperative delirium is a common complication of major surgery in elderly patients, but often remains undiagnosed. ${ }^{1}$ Also, delirium occurring after cardiac surgery is a common acute neurocognitive disorder bearing severe consequences for the patients. ${ }^{2,3}$ Previously, numerous poorly defined psychiatric disorders were identified following heart surgery - acute brain syndrome, post-cardiotomy syndrome, postoperative psychosis - that now lie under the common definition of post-cardiac surgery delirium. ${ }^{4,5}$ Delirium is defined using the Diagnostic Statistical Manual of
Correspondence: Katarzyna Kotfis Department of Anesthesiology, Intensive Therapy and Acute Intoxications, Pomeranian Medical University, Teaching Hospital No. 2, al. Powstańców Wielkopolskich 72, 70-I I I Szczecin, Poland Tel/fax +48 9I 466 II 44 Email katarzyna.kotfis@pum.edu.pl 
Mental Disorders (DSM) criteria as disturbance in attention accompanied by changes in awareness and cognition occurring acutely and having a fluctuating course. ${ }^{6}$

Depending on methodology, post-cardiac surgery delirium is diagnosed in 26\%-52\% of patients, with some studies reporting percentages as high as $70 . .^{3,7,8}$ In patients older than 60 years, the reported incidence is similar - between $30 \%$ and $52 \% .{ }^{9,10}$ With aging of populations worldwide and an increasing number of cardiac operations performed yearly, post-cardiac surgery delirium is a major epidemiologic and clinical problem. The consequences of delirium are long-lived and include increased mortality and morbidity, long-term cognitive dysfunction and memory loss, increased risk of falls, and decreased functional status. ${ }^{11,12}$ It is also important to identify risk factors, including exercise capacity prior to the operation, as postoperative delirium after cardiac surgery may be associated with poor exercise tolerance. ${ }^{13-15}$ Thorough knowledge of delirium incidence and the frequency of complications in certain patient populations, especially at the extremes of age, can help allocate resources and guide future research.

The pathophysiology of delirium is not fully elucidated, but a common pathway lies in neuroinflammation. This distressing syndrome can be regarded as a marker of cerebral decompensation in response to physiologic stressors such as organ failure, inflammation, infection, or drug effect. The use of cardiopulmonary bypass (CPB) in cardiac surgery leads to systemic inflammation with endothelial dysfunction and blood-brain barrier disruption causing neuroinflammation and activation of microglial cells. ${ }^{16,17}$ Only identification of patients at risk of developing delirium and its timely detection can reduce the impact of acute brain dysfunction and allow targeted interventions. ${ }^{18}$ A recent systematic review listed 11 strong risk factors including age, type of surgery, preoperative history of cognitive decline, previous psychiatric conditions, preoperative cerebrovascular disease, perioperative use of risperidone, postoperative oxygen saturation, mechanical ventilation time, blood product transfusion, occurrence of postoperative atrial fibrillation, and renal insufficiency. ${ }^{19}$

Multiple studies have reported risk factors for delirium after cardiac surgery, all highlighting advancing age as one of the major unmodifiable factors. Despite increased interest in delirium after cardiac surgery, few studies have focused on research specifically in elderly patients, with relatively low numbers of patients included in these studies. Therefore, the aim of this study was to analyze the prevalence of delirium in patients undergoing cardiac surgery, to identify preoperative, intraoperative, and postoperative risk factors of intensive care unit (ICU) delirium after cardiac surgery and to correlate ICU delirium after cardiac surgery with the length of hospital stay, length of stay (LOS) in the ICU, intubation time, and mortality in elderly ( $\geq 65$ years) and very elderly ( $\geq 80$ years) patients.

\section{Materials and methods}

We performed a retrospective cohort analysis of prospectively collected data from a register of the cardiac surgery department of a tertiary referral university hospital over three consecutive years from January 1st, 2014 until December 31st, 2016. From the general cohort of patients undergoing cardiac surgery at our department we extracted data regarding all elderly ( $\geq 65$ years) patients of both genders, as according to the World Health Organization, the chronologic age of 65 years was accepted by most developed countries in the world as a definition of "elderly" or older person. Out of the group of patients above 65 years, we further extracted data regarding very elderly patients ( $\geq 80$ years). The inclusion criterion was major cardiac operation performed under general anesthesia. The following exclusion criteria were used: known preoperative cognitive disorder (dementia, mild cognitive impairment, Mini Mental State Examination score below 24 points), psychiatric or mental disorder (epilepsy, schizophrenia, depression, and so on), and patients who died intraoperatively or within the first 6 hours after the procedure. The analysis was performed according to age in two groups: Group I included all patients with age equal to or above 65 years with and without delirium ( $\geq 65$ years delirium + vs delirium-) and Group II included all patients with age equal to or above 80 years with and without delirium ( $\geq 80$ years delirium+ vs delirium-).

\section{Data collection}

Evaluation of potential risk factors was based on the existing literature on the subject and the availability of the data in our database. Risk factors for postoperative delirium were divided into three categories - preoperative, intraoperative, and postoperative. The demographic and comorbid conditions (hypertension, coronary artery disease, congestive heart failure, dyslipidemia, thyroid disorders, arrhythmias, diabetes mellitus or impaired glucose tolerance, chronic obstructive pulmonary disease, renal disease [acute renal failure, renal insufficiency, chronic renal disease, end-stage renal disease], smoking prior to surgery), as well as risk factors were obtained from the medical records, an interview with the patient, and the preoperative anesthetic questionnaire. Data regarding postoperative outcome included intubation 
time, hospital and ICU LOS, and mortality. Both preoperative assessment and premedication, as well as intraoperative surgical and anesthetic approach were performed according to well-established local guidelines. Laboratory data results came from standard pre- and postoperative blood sampling. Cardiac procedures included all major operations performed under general anesthesia: coronary artery bypass grafting (CABG), valve/valves replacement and/or valve repair including transthoracic aortic valve implementation (TAVI), CABG plus valve replacement and/or valve repair, $\mathrm{CABG}$ with ascending aorta aneurysm surgery (AAA), or combined with valve procedures. All patients were anesthetized according to a local protocol, using a standard set of drugs and doses for each patient.

Induction of general anesthesia was performed intravenously using fentanyl $\left(3 \mu \mathrm{g} \mathrm{kg}^{-1}\right)$ and etomidate $0.2 \mathrm{mg} \mathrm{kg}^{-1}$, whereas pancuronium $0.5 \mathrm{mg} \mathrm{kg}^{-1}$ was used to facilitate tracheal intubation. Maintenance of general anesthesia was performed with sevoflurane $2-2.5 \mathrm{Vol} \%$ and additional doses of fentanyl (3-5 $\mathrm{g} \mathrm{kg}^{-1}$ ), with muscle relaxation maintained with additional doses of pancuronium. All patients received heparin $100 \mathrm{IU} \mathrm{kg}{ }^{-1}$ before CPB initiation, with additional heparin doses guided by the activated clotting time. After the procedure heparin action was reversed with protamine sulfate infusion. After the operation, each patient remained intubated and mechanically ventilated and was transferred to the postoperative cardiac ICU. For postoperative pain control, multimodal analgesia was used with intravenous morphine infusion and non-opioid analgesic administration (paracetamol or metamizole). Patients were sedated according to a local protocol with dexmedetomidine $\left(0.4-0.7 \mu \mathrm{g} \mathrm{kg}^{-1} \mathrm{~h}\right)$ and extubated in the postoperative ICU when they met extubation criteria.

Intraoperative data collected into the register included the length of operation, length of bypass time (if applicable), time of cross-clamping (if applicable), and estimated blood loss (postoperative drainage). The outcome data included postoperative complications (cerebral, renal, infectious, cardiac, pulmonary) as well as hospital LOS, ICU LOS, intubation time, and 30-day mortality.

\section{Delirium evaluation}

All patients were screened for the presence of delirium by ICU physicians, attending cardiac surgeons, and nurses according to Diagnostic Statistical Manual of Mental Disorders, fifth edition (DSM-5) criteria during the postoperative course with data regarding the first 5 days after the operation included in the analysis. The time frame of 5 days was chosen arbitrarily as it reflects both early postoperative delirium and the average hospitalization time in our institution. Standard delirium screening tools were not in use at the facility during data collection, and delirium diagnosis was based on clinical assessment and guided by standard criteria of DSM-5. ${ }^{6}$ In research studies, use of either International Statistical Classification of Diseases and Related Health Problems, 10th revision or DSM-5 criteria is recommended as the gold standard diagnostic criteria. ${ }^{6}$ According to DSM-5 diagnostic criteria for delirium were 1) disturbance in attention (ie, reduced ability to direct, focus, sustain, and shift attention) and awareness (reduced orientation to the environment);2) disturbance developed over a short period of time (hours to days), changed from baseline and fluctuated in severity during the course of a day; 3 ) additional disturbance in cognition (eg, memory deficit, disorientation, language, visuospatial ability, or perception). Moreover, criteria from 1 and 2 were not better explained by another preexisting, established, or evolving neurocognitive disorder or coma and there was no evidence from preexisting history, physical examination, or laboratory findings that the disturbance is a direct physiologic consequence of another medical condition, substance intoxication or exposure to toxin, substance withdrawal, or because of multiple etiologies. ${ }^{6}$ Initial delirium assessments were performed in the postoperative ICU by at least two health care professionals (doctor and/or nurse) with the patients sedated and undergoing mechanical ventilation and after the patient had been weaned from mechanical ventilation and extubated. Data regarding delirium were extracted from detailed nursing and medical staff records.

\section{Ethical concerns}

The study was performed in accordance with the Declaration of Helsinki. The study received a waiver from the Bioethical Committee of the Pomeranian Medical University in Szczecin, Poland, due to its retrospective character, decision no KB-0012/79/01/18. Nevertheless, written informed consent for surgery and anesthesia was obtained before surgery from each patient as part of routine preoperative evaluation and included consent for data collection. Patient consent to review their medical records was waived by the Bioethical Committee. To ensure patient confidentiality the analysis was performed on de-identified data.

\section{Statistical analysis}

The descriptive statistics were performed to characterize the study population. Continuous variables were presented as means with SD. We presented categorical variables as 
proportions and compared using chi-square test. The incidence of delirium was calculated for each age group, namely for all patients $\geq 65$ years $\geq 80$ years and chi-square test was used for comparison between both groups (delirium vs no delirium) in each age category. We analyzed risk factors in both groups (delirium vs no delirium) for both age categories. The normality of distribution was checked using a Shapiro-Wilk test. The uniformity of variance was assessed using a Levene test. Data analysis for the two groups was performed using a Mann-Whitney $U$-test. We used univariate logistic regression analysis for each variable and presented it as odds ratios (OR) with 95\% CIs. Multivariable logistic regression analysis was performed for risk factors that were significant in univariate analysis and presented with narrow CIs. $p$-value of $<0.05$ was regarded as statistically significant. All data were analyzed using licensed software Statistica 12 (StatSoft, Inc., Tulsa, OK, USA), license no JPZP602C295824AR-V.

\section{Results}

We analyzed a cohort of 3,178 patients and included 1,797 (56.55\%) patients $\geq 65$ years in the final analysis, out of which we extracted data for patients $\geq 80$ years -230 (7.24\%). Delirium was diagnosed in $21.4 \%(384 / 1,797)$ patients aged $\geq 65$ years, and in $33.5 \%$ (77/230) of octogenarians.

\section{Preoperative characteristics}

Demographic data and concomitant diseases are included in Table 1 for both age categories. The percentage of females and males did not differ between the two groups in both age categories, and the majority of patients were males. The most common comorbid conditions were hypertension, diabetes, congestive heart failure, and extracardiac arteriopathy. Patients aged $\geq 65$ with delirium were older $(73.47 \pm 6.08$ vs $71.99 \pm 5.52, p<0.001)$, were more often diabetic $(53.65 \%$ vs $43.74 \%, p<0.001)$, more often presented with internal carotid artery stenosis $(11.72 \%$ vs $7.93 \%, p=0.019)$, chronic

Table I Patients' baseline characteristics for patients $\geq 65$ years and $\geq 80$ years of age with and without delirium

\begin{tabular}{|c|c|c|c|c|c|c|}
\hline Variable & $\begin{array}{l}\text { No delirium } \\
\geq 65 \text { years } \\
(n=I, 4 I 3)\end{array}$ & $\begin{array}{l}\text { Delirium } \\
\geq 65 \text { years } \\
(n=384)\end{array}$ & p-value & $\begin{array}{l}\text { No delirium } \\
\geq 80 \text { years } \\
(n=153)\end{array}$ & $\begin{array}{l}\text { Delirium } \\
\geq 80 \text { years } \\
(n=77)\end{array}$ & $p$-value \\
\hline \multicolumn{7}{|l|}{ Demographic data } \\
\hline Age (years), mean $\pm S D$ & $71.99 \pm 5.52$ & $73.47 \pm 6.08$ & $<0.001$ & $82.56 \pm 2.64$ & $82.44 \pm 2.59$ & NS \\
\hline Gender, male, n (\%) & $905(64.05)$ & $256(66.67)$ & NS & $85(55.56)$ & $52(67.53)$ & NS \\
\hline Smoking, n (\%) & $302(21.37)$ & $84(21.88)$ & NS & $15(9.80)$ & $6(7.79)$ & NS \\
\hline $\mathrm{BMI}$, mean $\pm \mathrm{SD}, \mathrm{kg} / \mathrm{m}^{2}$ & $28.6 I \pm 4.37$ & $28.7 I \pm 4.34$ & NS & $28.07 \pm 4.18$ & $28.21 \pm 3.48$ & NS \\
\hline \multicolumn{7}{|l|}{ Concomitant diseases } \\
\hline \multicolumn{7}{|l|}{ Ischemic heart disease, n (\%) } \\
\hline CCS I & $68(5.57)$ & $15(4.36)$ & NS & $5(4.00)$ & $5(6.94)$ & \\
\hline CCS II & $406(33.28)$ & $125(36.34)$ & & $34(27.20)$ & $27(37.50)$ & \\
\hline CCS III & $489(40.08)$ & $124(36.05)$ & & $52(4 I .60)$ & $18(25.00)$ & NS \\
\hline CCS IV & $257(21.07)$ & $80(23.26)$ & & $34(27.20)$ & $22(30.56)$ & \\
\hline Diabetes, n (\%) & $618(43.74)$ & $206(53.65)$ & $<0.001$ & $69(45.10)$ & $40(51.95)$ & NS \\
\hline Glucose intolerance, n (\%) & $63(4.46)$ & $9(2.34)$ & NS & $9(5.88)$ & $3(3.90)$ & NS \\
\hline TIA, n (\%) & $23(1.63)$ & $3(0.78)$ & NS & $6(3.92)$ & $0(0.00)$ & NS \\
\hline Stroke, n (\%) & $77(5.45)$ & $26(6.77)$ & NS & $10(6.54)$ & $9(11.69)$ & NS \\
\hline Arterial hypertension, n (\%) & $\mathrm{I}, 04 \mathrm{I}(73.67)$ & $294(76.56)$ & NS & $113(73.86)$ & $63(81.82)$ & NS \\
\hline AF paroxysmal, n (\%) & $113(8.00)$ & $37(9.64)$ & NS & $15(9.80)$ & $6(7.79)$ & NS \\
\hline AF persistent, $n(\%)$ & $136(9.62)$ & $38(9.90)$ & NS & $14(9.15)$ & $8(10.39)$ & NS \\
\hline ICA stenosis, n (\%) & $112(7.93)$ & $45(11.72)$ & 0.019 & $20(13.07)$ & $9(11.69)$ & NS \\
\hline COPD, n (\%) & $98(7.25)$ & $36(9.60)$ & NS & $15(10.34)$ & $3(4.05)$ & NS \\
\hline Chronic renal failure, $n(\%)$ & $132(9.34)$ & $52(13.54)$ & 0.016 & $30(19.61)$ & II (14.29) & NS \\
\hline Acute renal failure, $\mathrm{n}(\%)$ & $12(0.85)$ & $7(1.82)$ & NS & $3(1.96)$ & $2(2.60)$ & NS \\
\hline Ejection fraction $<30 \%$, n (\%) & $87(6.16)$ & $38(9.90)$ & 0.011 & $9(5.88)$ & $4(5.19)$ & NS \\
\hline \multicolumn{7}{|l|}{ Congestive heart failure, $\mathrm{n}(\%)$} \\
\hline NYHA I & $53(6.36)$ & $4(1.55)$ & 0.006 & $2(1.68)$ & $2(3.57)$ & \\
\hline NYHA II & $390(46.82)$ & III (43.02) & & $54(45.38)$ & $26(46.43)$ & \\
\hline NYHA III & $326(39.38)$ & $119(46.12)$ & & $54(45.38)$ & $26(46.43)$ & NS \\
\hline NYHA IV & $62(7.44)$ & $24(9.30)$ & & $9(7.56)$ & $2(3.57)$ & \\
\hline Extracardiac arteriopathy, n (\%) & $232(17.07)$ & $98(25.99)$ & $<0.001$ & $26(17.93)$ & $17(22.67)$ & NS \\
\hline
\end{tabular}

Abbreviations: AF, atrial fibrillation; BMI, body mass index $\left(\mathrm{kg} \mathrm{m}^{-2}\right)$; CCS, Canadian Cardiovascular Society; ICA, internal carotid artery; n, number of patients; NS, not significant; NYHA, New York Heart Association; TIA, transient ischemic attack. 
renal failure (13.54 vs 9.34, 0.016), ejection fraction $<30 \%$ $(9.90 \%$ vs $6.16 \%, 0.011)$, and extracardiac arteriopathy ( $25.99 \%$ vs $17.07 \%, p<0.001)$, than their counterparts without delirium. There were no statistically significant differences between patients $\geq 80$ years of age in groups with and without delirium.

\section{Operative and postoperative characteristics}

Operative and postoperative patient characteristics are shown in Table 2 . In patients aged $\geq 65$ who developed postoperative delirium, the operative risk was higher when calculated using
EuroScore Logistic $(12.66 \pm 12.22$ vs 9.64 $\pm 10.51, p<0.001)$ or EuroScore Logistic 2 scale (4.58 \pm 5.49 vs 3.53 \pm 5.01 , $p<0.001)$. In this group of patients more complex surgery was associated with delirium (CABG + valve: $27.605 \%$ vs $17.34 \%, p<0.001)$. When analyzing intraoperative factors, for patients aged $\geq 65$ with delirium, the CPB time was longer ( $71.13 \pm 31.23$ vs $66.94 \pm 50.40 \mathrm{~min}, p=0.009$ ) and also the aortic cross-clamping time was longer than in nondelirious patients $(48.14 \pm 25.78$ vs $44.02 \pm 24.46 \mathrm{~min}$, $p=0.003$ ). Postoperative glomerular filtration rate (GFR) was lower in patients older than 65 years with delirium $(45.54 \pm 24.37$ vs $57.27 \pm 23.71, p<0.001)$ and creatinine

Table 2 Patients' operative and postoperative characteristics

\begin{tabular}{|c|c|c|c|c|c|c|}
\hline Variable & $\begin{array}{l}\text { No delirium } \\
\geq 65 \text { years } \\
(n=1,413)\end{array}$ & $\begin{array}{l}\text { Delirium } \\
\geq 65 \text { years } \\
(n=384)\end{array}$ & $p$-value & $\begin{array}{l}\text { No delirium } \\
\geq 80 \text { years } \\
(n=I 53)\end{array}$ & $\begin{array}{l}\text { Delirium } \\
\geq 80 \text { years } \\
(n=77)\end{array}$ & p-value \\
\hline \multicolumn{7}{|l|}{ Operative risk } \\
\hline EuroScore logistic, mean $\pm S D$ & $9.64 \pm 10.51$ & $12.66 \pm 12.22$ & $<0.001$ & $17.71 \pm 13.47$ & $18.46 \pm 13.44$ & NS \\
\hline EuroScore logistic 2 , mean $\pm S D$ & $3.53 \pm 5.01$ & $4.58 \pm 5.49$ & $<0.001$ & $4.64 \pm 4.56$ & $5.21 \pm 5.73$ & NS \\
\hline \multicolumn{7}{|l|}{ Operation type } \\
\hline Isolated CABG, n (\%) & $880(62.28)$ & $211(54.95)$ & 0.009 & $77(50.33)$ & $45(58.44)$ & NS \\
\hline CABG + valve, n (\%) & $245(17.34)$ & $106(27.60)$ & $<0.001$ & $34(22.22)$ & $20(25.97)$ & NS \\
\hline CABG + AAA, n (\%) & $20(1.42)$ & $6(1.56)$ & NS & $0(0.00)$ & I (I.30) & NS \\
\hline TAVI, n (\%) & $24(1.70)$ & $4(1.04)$ & NS & $18(11.76)$ & $2(2.60)$ & 0.019 \\
\hline Aortic aneurysm, n (\%) & $66(4.76)$ & $16(4.17)$ & NS & $4(2.6 I)$ & $3(3.90)$ & NS \\
\hline Valve, n (\%) & $178(12.60)$ & 41 (10.68) & NS & $20(13.07)$ & $6(7.79)$ & NS \\
\hline \multicolumn{7}{|l|}{ Case priority } \\
\hline Planned, n (\%) & $\mathrm{I}, 028(72.75)$ & $279(72.66)$ & & $94(61.44)$ & $48(62.34)$ & \\
\hline Urgent, n (\%) & $311(22.01)$ & $81(21.09)$ & NS & $52(33.99)$ & $20(25.97)$ & NS \\
\hline Emergent, n (\%) & $74(5.24)$ & $24(6.1)$ & & $7(4.58)$ & $9(11.69)$ & \\
\hline \multicolumn{7}{|l|}{ Intraoperative data } \\
\hline $\mathrm{CPB}$ time $(\min )$, mean $\pm \mathrm{SD}$ & $66.94 \pm 50.40$ & $71.13 \pm 31.23$ & 0.009 & $64.40 \pm 27.91$ & $68.30 \pm 28.82$ & NS \\
\hline Aortic cross-clamping (min), mean $\pm \mathrm{SD}$ & $44.02 \pm 24.46$ & $48.14 \pm 25.78$ & 0.003 & $44.61 \pm 23.83$ & $46.72 \pm 24.13$ & NS \\
\hline Reperfusion time (min), mean \pm SD & $16.58 \pm 9.68$ & $16.97 \pm 9.05$ & NS & $14.96 \pm 6.98$ & $16.81 \pm 11.02$ & NS \\
\hline \multicolumn{7}{|l|}{ Postoperative data } \\
\hline Drainage $(\mathrm{mL})$ & $485 \pm 528$ & $529 \pm 477$ & 0.04 & $438 \pm 416$ & $535 \pm 543$ & NS \\
\hline Highest creatinine (mg/dL) & $\mathrm{I} .4 \mathrm{I} \pm 0.87$ & $1.86 \pm 1.29$ & $<0.001$ & $1.63 \pm 0.84$ & $1.86 \pm 1.08$ & NS \\
\hline Lowest GFR (mL/min/l.73 m²) & $57.27 \pm 23.71$ & $45.54 \pm 24.37$ & $<0.001$ & $43.06 \pm 18.52$ & $39.78 \pm 20.40$ & NS \\
\hline \multicolumn{7}{|l|}{ Transfusions } \\
\hline PRBCs (mL) & $954 \pm 852$ & $1,047 \pm 847$ & 0.03 & $1,024 \pm 879$ & $1,126 \pm 904$ & NS \\
\hline FFP $(m L)$ & $929 \pm 715$ & $927 \pm 504$ & NS & $872 \pm 411$ & $932 \pm 563$ & NS \\
\hline Platelets (mL) & $353 \pm 182$ & $366 \pm 153$ & NS & $339 \pm 147$ & $38 I \pm 184$ & NS \\
\hline \multicolumn{7}{|l|}{ Postoperative complications } \\
\hline Stroke, n (\%) & $53(3.75)$ & $19(4.95)$ & NS & $12(7.84)$ & $0(0)$ & 0.027 \\
\hline Atrial fibrillation, n (\%) & 324 (22.93) & I3I (34.II) & $<0.001$ & $42(27.45)$ & $30(38.96)$ & NS \\
\hline Low cardiac output, n (\%) & $56(3.96)$ & $14(3.65)$ & NS & $4(2.6 I)$ & $2(2.60)$ & NS \\
\hline Respiratory failure, n (\%) & $133(9.41)$ & $84(21.88)$ & $<0.001$ & $24(15.69)$ & $17(22.08)$ & NS \\
\hline Pneumonia, n (\%) & $42(2.97)$ & 45 (II.72) & $<0.001$ & $6(3.92)$ & 10 (12.99) & 0.011 \\
\hline Acute kidney injury, n (\%) & $39(2.76)$ & $25(6.5 I)$ & $<0.001$ & $9(5.88)$ & $7(9.09)$ & NS \\
\hline AKI with CRRT, n (\%) & $160(11.31)$ & $93(24.22)$ & $<0.001$ & $28(18.30)$ & $18(23.38)$ & NS \\
\hline Sepsis, n (\%) & $7(0.5)$ & I (0.26) & NS & I (0.65) & $6(7.79)$ & NS \\
\hline
\end{tabular}

Abbreviations: AAA, ascending aorta aneurysm; AKI, acute kidney injury; CABG, coronary artery bypass grafting; GFR, glomerular filtration rate; CPB, cardiopulmonary bypass; CRRT, continuous renal replacement therapy; FFP, fresh frozen plasma; n, number of patients; NS, not significant; PRBCs, packed red blood cells; TAVI, transcatheter aortic valve implantation. 
was higher $(1.86 \pm 1.29$ vs $1.41 \pm 0.87, p<0.001)$. When looking at postoperative complications in elderly patients $(\geq 65$ years) with delirium, atrial fibrillation $(34.11 \%$ vs $22.93 \%$, $p<0.001)$, respiratory failure $(21.88 \%$ vs $9.41 \%, p<0.001)$, pneumonia $(11.72 \%$ vs $2.97 \%, p<0.001)$, acute kidney injury (6.51\% vs $2.76 \%, p<0.001)$, and acute kidney injury requiring hemofiltration $(24.22 \%$ vs $11.31 \%, p<0.001)$ occurred more often than in patients without delirium after cardiac surgery. The differences between patients with and without delirium are less evident in octogenarians.

\section{Postoperative outcome}

Data regarding postoperative outcome are shown in Table 3 for both age groups. Mortality during 30 postoperative days did not differ between patients with and without delirium in subgroups aged over 65 years and over 80 years. Both elderly and very elderly patients had longer hospital LOS if they developed postoperative delirium, $13.91 \pm 14.8 \mathrm{vs}$ $9.39 \pm 8.09, p<0.001$ and $16.75 \pm 20.46$ vs $10.56 \pm 10.21$ days, $p=0.003$, respectively. The LOS in the cardiac surgery ICU was longer for elderly patients with delirium (3.10 $\pm 6.69 \mathrm{vs}$ $2.05 \pm 5.21$ days, $p<0.001)$. Intubation time was longer in patients above 65 years with delirium $(1,342 \pm 2,731 \mathrm{~min}$ vs $1,244 \pm 2,756 \mathrm{~min}, p=0.002$ ).

\section{Univariate analysis}

The results of univariate analysis are shown in Table 4. Patients who developed post-cardiac surgery delirium had more advanced age $(p<0.001)$, lower ejection fraction $(p=0.001)$, were diabetic $(p=0.001)$, had internal carotid artery stenosis $(p=0.02)$, chronic renal failure $(p=0.017)$, heart failure $(p<0.001)$, higher operative risk $(p=0.001)$, higher creatinine $(p=0.003)$, and lower GFR $(p<0.001)$ on admission. The only operative factor was longer aortic crossclamping time $(p=0.005)$. Postoperative risk factors included higher creatinine $(p<0.001)$, lower GFR $(p<0.001)$, pneumonia $(p<0.001)$, respiratory failure $(p<0.001)$, acute renal failure requiring continuous renal replacement therapy $(p=0.001)$, and longer ICU $(p=0.003)$ and hospital LOS $(p<0.001)$.

\section{Multivariable analysis}

Results of the multivariable logistic regression analysis are presented in Table 5, and only statistically significant results are included. Eight independent risk factors of post-cardiac surgery delirium were identified. When analyzing preoperative factors in patients above 65 years, age (OR 1.036, $p=0.002$ ), low ejection fraction (OR $1.634, p=0.035)$, diabetes (1.346, $p=0.019$ ), and extracardiac arteriopathy (OR 1.564, $p=0.007$ ) were found to be independent predictors of postcardiac surgery delirium in elderly patients. Postoperative risk factors of developing delirium for patients above 65 years were atrial fibrillation $(1.563, p=0.001)$, postoperative pneumonia (OR 1.896, $p=0.022$ ), elevated postoperative creatinine (OR 1.384, $p=0.004$ ), and prolonged hospital LOS (OR 1.019, $p=0.009$ ). For octogenarians, no significant predictors of postoperative delirium occurrence were found.

\section{Discussion}

Post-cardiac surgery delirium must be acknowledged as a major postoperative neurologic complication. The incidence of delirium in the elderly patients in our study was relatively low, with one in five $(21.4 \%)$ patients above 65 years developing delirium and one in three (33.5\%) above the age of 80 years suffering from this type of acute brain dysfunction.

The analysis of outcome showed, interestingly, lower mortality during 30 postoperative days in patients with delirium, both above 65 years (5.73\% vs $7.15 \%)$ and above 80 years $(7.79 \%$ vs $9.80 \%)$, although this was not statistically significant. Patients above 65 years who developed delirium had longer hospital LOS $(p<0.001)$, longer ICU stay $(p<0.001)$, and longer intubation time $(p=0.002)$.

Results of the multivariable analysis of our data identified eight independent risk factors of post-cardiac surgery delirium. In patients above 65 years, age (OR 1.036, $p=0.002$ ), low ejection fraction (OR $1.634, p=0.035)$, diabetes

Table 3 Outcome data for patients $\geq 65$ and $\geq 80$ years of age with and without delirium

\begin{tabular}{|c|c|c|c|c|c|c|}
\hline Outcome data & $\begin{array}{l}\text { No delirium } \\
\geq 65 \text { years }\end{array}$ & $\begin{array}{l}\text { Delirium } \\
\geq 65 \text { years }\end{array}$ & $p$-value & $\begin{array}{l}\text { No delirium } \\
\geq 80 \text { years }\end{array}$ & $\begin{array}{l}\text { Delirium } \\
\geq 80 \text { years }\end{array}$ & $p$-value \\
\hline Mortality 30 days, n (\%) & $101(7.15)$ & $22(5.73)$ & NS & $15(9.80)$ & $6(7.79)$ & NS \\
\hline Hospital LOS, days, mean \pm SD & $9.39 \pm 8.09$ & $13.91 \pm 14.8$ & $<0.001$ & $10.56 \pm 10.21$ & $16.75 \pm 20.46$ & 0.003 \\
\hline ICU LOS, days, mean \pm SD & $2.05 \pm 5.21$ & $3.10 \pm 6.69$ & $<0.001$ & $2.56 \pm 6.11$ & $1.94 \pm 3.72$ & NS \\
\hline Intubation time $(\min )$, mean $\pm S D$ & $\mathrm{I}, 244 \pm 2,756$ & $\mathrm{I}, 342 \pm 2,73 \mathrm{I}$ & 0.002 & $\mathrm{I}, 945 \pm 4,778$ & $\mathrm{I}, 030 \pm 864$ & NS \\
\hline
\end{tabular}

Abbreviations: ICU, intensive care unit; LOS, length of stay; NS, not significant. 
Table 4 Univariate regression analysis for patients with delirium $\geq 65$ years

\begin{tabular}{|c|c|c|c|}
\hline $\begin{array}{l}\text { Variables associated with } \\
\text { delirium in patients } \geq 65 \text { years }\end{array}$ & $p$-value & OR & $95 \% \mathrm{Cl}$ \\
\hline \multicolumn{4}{|l|}{ Demographic data } \\
\hline Age (years) & $<0.001$ & 1.046 & $1.026-1.066$ \\
\hline BMI $\left(\mathrm{kg} \mathrm{m}^{-2}\right)$ & NS & 1.005 & $0.98-1.031$ \\
\hline Male gender, n (\%) & NS & 1.123 & $0.885-1.425$ \\
\hline Smoking, n (\%) & NS & $0.97 I$ & $0.739-1.276$ \\
\hline Smoking (years) & NS & 1.006 & $0.985-1.0128$ \\
\hline \multicolumn{4}{|l|}{$\mathrm{BMI}$} \\
\hline Normal & NS & 0.982 & $0.7 \mid 3-1.354$ \\
\hline Overweight & NS & 1.177 & $0.867-1.598$ \\
\hline Obese & NS & 1.018 & $0.739-1.403$ \\
\hline \multicolumn{4}{|l|}{ Case priority } \\
\hline Planned & NS & 0.837 & $0.5|8-| .35 \mid$ \\
\hline Urgent & NS & 0.960 & $0.727-1.267$ \\
\hline Emergency & NS & 1.195 & $0.740-1.929$ \\
\hline \multicolumn{4}{|l|}{$\mathrm{EF} \%$} \\
\hline$\geq 50 \%$ & 0.001 & 0.497 & $0.328-0.753$ \\
\hline $50 \%-30 \%$ & 0.001 & 1.502 & $1.184-1.904$ \\
\hline$<30 \%$ & 0.001 & 2.013 & $1.329-3.049$ \\
\hline \multicolumn{4}{|l|}{ Concomitant diseases } \\
\hline \multicolumn{4}{|l|}{ Ischemic heart disease } \\
\hline CCSI & NS & 0.709 & $0.384-1.308$ \\
\hline CCS ॥ & NS & 1.396 & $0.77 I-2.528$ \\
\hline CCS III & NS & 1.150 & $0.635-2.080$ \\
\hline CCS IV & NS & 1.411 & $0.765-2.605$ \\
\hline Diabetes & 0.001 & 1.489 & I.I87-I.867 \\
\hline Glucose intolerance & NS & 0.514 & $0.253-1.044$ \\
\hline Stroke & NS & 1.260 & $0.796-1.995$ \\
\hline Arterial hypertension & NS & 1.167 & $0.896-1.520$ \\
\hline AF paroxysmal & NS & 1.227 & $0.831-1.811$ \\
\hline AF persistent & NS & 1.031 & $0.706-1.506$ \\
\hline Internal carotid artery stenosis & 0.02 & 1.542 & $1.069-2.223$ \\
\hline COPD & NS & 1.358 & $0.910-2.026$ \\
\hline Chronic renal failure & 0.017 & 1.520 & $1.079-2.142$ \\
\hline Acute renal failure & NS & 2.168 & $0.848-5.544$ \\
\hline Heart failure & $<0.001$ & 1.556 & $1.227-1.974$ \\
\hline \multicolumn{4}{|l|}{ Congestive heart failure } \\
\hline NYHA I & 0.004 & 0.195 & $0.064-0.598$ \\
\hline NYHA II & 0.012 & 3.771 & $1.336-10.648$ \\
\hline NYHA III & 0.003 & 4.807 & $1.703-13.570$ \\
\hline NYHA IV & 0.004 & 5.129 & $1.673-15.723$ \\
\hline Extracardiac arteriopathy & $<0.001$ & 1.706 & $1.302-2.235$ \\
\hline \multicolumn{4}{|l|}{ Operative risk } \\
\hline EuroScore logistic & $<0.001$ & 1.022 & $1.013-1.031$ \\
\hline EuroScore logistic 2 & 0.001 & 1.034 & $1.014-1.054$ \\
\hline \multicolumn{4}{|l|}{ Preoperative factors } \\
\hline Hemoglobin at admission & NS & 1.004 & $0.998-1.009$ \\
\hline Creatinine $(\mathrm{mg} / \mathrm{dL})$ at admission & 0.003 & 1.002 & $1.001-1.003$ \\
\hline GFR at admission $\left(\mathrm{mL} / \mathrm{min} / \mathrm{l} .73 \mathrm{~m}^{2}\right)$ & $<0.001$ & 0.987 & $0.982-0.993$ \\
\hline \multicolumn{4}{|l|}{ Intraoperative factors } \\
\hline Cardiopulmonary bypass time & NS & 1.002 & $0.999-1.004$ \\
\hline Aortic cross-clamping time & 0.005 & 1.006 & $1.002-1.011$ \\
\hline Reperfusion time & NS & 1.004 & $0.993-1.016$ \\
\hline Intraoperative hemofiltration & NS & 1.000 & 1.000 \\
\hline \multicolumn{4}{|l|}{ Operation type } \\
\hline Isolated CABG, $n(\%)$ & NS & 1.439 & $0.494-4.191$ \\
\hline CABG + valve, $n(\%)$ & NS & 2.596 & $0.879-7.665$ \\
\hline
\end{tabular}

(Continued)
Table 4 (Continued)

\begin{tabular}{|c|c|c|c|}
\hline $\begin{array}{l}\text { Variables associated with } \\
\text { delirium in patients } \geq 65 \text { years }\end{array}$ & $p$-value & OR & $95 \% \mathrm{Cl}$ \\
\hline$\overline{C A B G}+\mathrm{AAA}, \mathrm{n}(\%)$ & NS & 1.800 & $0.445-7.281$ \\
\hline TAVI, n (\%) & NS & 0.385 & $0.130-1.137$ \\
\hline Aortic aneurysm, n (\%) & NS & 1.455 & $0.442-4.787$ \\
\hline Valve, n (\%) & NS & 1.382 & $0.455-4.200$ \\
\hline \multicolumn{4}{|l|}{ Postoperative factors } \\
\hline Intubation time & NS & 1.000 & 1.000 \\
\hline Drainage & NS & 1.000 & 1.000 \\
\hline $\begin{array}{l}\text { Creatinine }(\mathrm{mg} / \mathrm{dL}) \text { highest } \\
\text { postoperative }\end{array}$ & $<0.001$ & 1.004 & $1.003-1.005$ \\
\hline $\begin{array}{l}\text { GFR }\left(\mathrm{mL} / \mathrm{min} / 1.73 \mathrm{~m}^{2}\right) \text { lowest } \\
\text { postoperative }\end{array}$ & $<0.001$ & 0.980 & $0.975-0.984$ \\
\hline Packed red blood cells transfusion & NS & 1.000 & $0.997-1.002$ \\
\hline Fresh frozen plasma transfusion & NS & 0.999 & $0.997-1.002$ \\
\hline Platelets transfusion & NS & 1.000 & $0.999-1.001$ \\
\hline \multicolumn{4}{|l|}{ Postoperative complications } \\
\hline Stroke & NS & 1.336 & $0.78 \mathrm{I}-2.285$ \\
\hline Low cardiac output & NS & 0.917 & $0.505-1.665$ \\
\hline Pneumonia & $<0.001$ & 4.333 & $2.799-6.708$ \\
\hline Respiratory failure & $<0.001$ & 2.695 & $1.995-3.640$ \\
\hline Acute renal failure & 0.001 & 2.453 & $1.465-4.108$ \\
\hline$A K I$ requiring CRRT & $<0.001$ & 2.503 & $1.88 \mathrm{I}-3.330$ \\
\hline Sepsis & NS & 0.524 & $0.064-4.276$ \\
\hline \multicolumn{4}{|l|}{ Outcome data } \\
\hline Hospital LOS (days) & $<0.001$ & 1.039 & $1.027-1.050$ \\
\hline ICU LOS (days) & 0.003 & 1.028 & $1.009-1.046$ \\
\hline Mortality 30 days, $\mathrm{n}(\%)$ & NS & 0.789 & $0.49 I-1.270$ \\
\hline
\end{tabular}

Abbreviations: AAA, ascending aorta aneurysm; AF, atrial fibrillation; $A K I$, acute kidney injury; BMI, body mass index; CABG, coronary artery bypass grafting; CCS, Canadian Cardiovascular Society; CPB, cardiopulmonary bypass; CRRT, continuous renal replacement therapy; EF, ejection fraction; GFR, glomerular filtration rate; ICU, intensive care unit; LOS, length of stay; NS, not significant; NYHA, New York Heart Association; OR, odds ratio; TAVI, transcatheter aortic valve implantation.

(1.346, $p=0.019)$, and extracardiac arteriopathy (OR 1.564, $p=0.007)$ were found to be independent predictors of postcardiac surgery delirium. Risk factors identified in the postoperative period in this age group were atrial fibrillation (1.563, $p=0.001$ ), postoperative pneumonia (OR $1.896, p=0.022$ ), elevated postoperative creatinine (OR 1.384, $p=0.004$ ), and prolonged hospital LOS (OR 1.019, $p=0.009$ ). For patients above 80 years, no significant predictors of post-cardiac surgery delirium were identified.

The incidence of delirium in our dataset is similar to results obtained from other studies concentrating on cardiac surgery; however, it is still lower than in the general ICU setting ${ }^{2,9,10,20,21}$ or after other types of major surgery. ${ }^{22}$ This may be related to factors not measured such as postoperative ICU design, patient preparation prior to surgery, or population differences in different geographical settings around the world. To our knowledge this is the first analysis reporting data specifically on elderly and very elderly patients. Moreover, this is the largest dataset reporting an analysis of the Polish population. 
Table 5 Multivariable regression analysis of patients aged $\geq 65$ years and $\geq 80$ years with delirium

\begin{tabular}{|c|c|c|c|c|c|c|}
\hline Variable & $\begin{array}{l}\text { OR, delirium } \\
\geq 65 \text { years }\end{array}$ & $95 \% \mathrm{Cl}$ & $p$-value & $\begin{array}{l}\text { OR, delirium } \\
\geq 80 \text { years }\end{array}$ & $95 \% \mathrm{Cl}$ & $p$-value \\
\hline \multicolumn{7}{|l|}{ Preoperative factors } \\
\hline Age & 1.036 & $1.012-1.059$ & 0.002 & 1.036 & $0.885-1.203$ & NS \\
\hline Ejection fraction $<30 \%$ & 1.634 & $1.035-2.578$ & 0.035 & 0.907 & $0.214-3.844$ & NS \\
\hline Diabetes & 1.346 & $1.049-1.726$ & 0.019 & 1.299 & $0.685-2.463$ & NS \\
\hline Extracardiac arteriopathy & 1.564 & $1.128-2.168$ & 0.007 & 1.458 & $0.618-3.438$ & NS \\
\hline \multicolumn{7}{|l|}{ Postoperative factors } \\
\hline Atrial fibrillation & 1.563 & I.195-2.046 & 0.001 & 1.463 & $0.746-2.870$ & NS \\
\hline Pneumonia & 1.896 & I.097-3.278 & 0.022 & 1.852 & $0.47 \mid-7.283$ & NS \\
\hline Postoperative creatinine (max) & 1.384 & $1.109-1.726$ & 0.004 & 1.096 & $0.640-1.877$ & NS \\
\hline Hospital LOS & 1.019 & $1.005-1.034$ & 0.009 & 1.027 & $0.996-1.059$ & NS \\
\hline
\end{tabular}

Abbreviations: LOS, length of stay; NS, not significant; OR, odds ratio.

Advanced age is a known predictor of delirium occurrence after cardiac surgery and in the general population. ${ }^{10,23}$ Our analysis showed that patients with delirium were, on average, 1.48 years older than patients without delirium (in the group above 65 years). The results of our study show that the negative consequences of delirium were evident in patients as young as 65 years. The differences in postoperative outcome were worse in delirious patients above the age of 65 , but not above the age of 80 . Interestingly, there were no independent risk factors identified for octogenarians. This may indicate that above a certain age the outcome and risks are similar whether patients develop delirium or not. This may be important information for the decision process on how to allocate resources.

Our data confirm the findings of previous studies. Norkiene et al reported only duration of postoperative mechanical ventilation and ICU stay to be independently associated with postoperative delirium in cardiac surgery. ${ }^{24}$ Other authors have also confirmed, as we have reported in our study, that patients with postoperative delirium have longer hospitalization times and a higher incidence of atrial fibrillation. ${ }^{25}$ In contrast to our study, Gosselt et al, in a systematic review, showed lack of evidence of an association of post-cardiac surgery delirium with gender, education, CPB duration, preexisting cardiac disease, preoperative heart failure. ${ }^{19}$ However, out of 11 strong risk factors reported by Gosselt et al, only age and postoperative renal failure were confirmed by our results. ${ }^{19}$ Elevated postoperative creatinine indicates an association between delirium and renal function, especially in the light of a high number of hypertension and diabetes induced nephropathies in this population. Moreover, diabetes itself turned out to be an independent risk factor. We did not find differences between isolated $\mathrm{CABG}$ and complex CABG with valve repair or replacement, which is also in contrast to other studies. ${ }^{26}$
Different mechanisms of neuropsychologic distress after cardiac surgery have been discussed, focusing on generalized inflammatory response, especially triggered by $\mathrm{CPB}$ or introduction of air microembolism during open heart surgery. ${ }^{27}$ Although not entirely proven, a number of hypotheses exist to explain the increased incidence and risk of post-cardiac surgery delirium in elderly patients. ${ }^{28}$ Some authors correlate it with more advanced atherosclerosis and endothelial dysfunction resulting in increased risk of cerebral microembolization. ${ }^{29}$ According to Lei et al, lower preoperative regional cerebral oxygen saturation may be associated with increased post-cardiac surgery delirium rates in patients $>60$ years..$^{30}$ Many elderly patients present with depleted cholinergic reserves and respond abnormally to anticholinergic medications commonly used in the perioperative period and during general anesthesia. ${ }^{17}$

Moreover, many authors underline that development of delirium in cardiac surgery is related to a complex interplay between predisposing factors, disease-associated elements (ie, atherosclerosis, inflammation), factors related to the operative technique, but also to some environmental cofactors (ICU design, noise, bright light, and so on).$^{31}$ Many of these factors, including health care professionals-associated elements (patient-nurse ratio, stress, excessive workload, professional burnout), are generally not measured in clinical studies, including our analysis, but seem to have an important influence on delirium development and should be taken into account. ${ }^{32}$

This study is not without limitations. First, this is a retrospective analysis of prospectively collected data, so some elements were not available in the dataset and the authors are unable to retrieve them. Second, due to the retrospective character of the analysis we were unable to identify patients with subsyndromal delirium, reported by other authors as a common occurrence with long-term cognitive consequences. Third, 
we did not use a validated screening tool to detect delirium. We decided to use the DSM- 5 criteria as the gold standard for delirium monitoring. The American College of Critical Care Medicine recommends screening using the Confusion Assessment Method for the Intensive Care Unit or Intensive Care Delirium Screening Checklist as the two tools validated for the critical care setting. ${ }^{33}$ However, these tools were not available in Polish until October 2015. Delirium assessments occurred throughout the day and the data from the whole 24-hour period was included in the daily summary chart. If the assessment was performed once or twice a day as in other studies (with a dedicated tool-based assessment protocol), it would have been missed and the prevalence of delirium would have been underestimated. Screening is still debated as it brings a lot of burden and stress to the team and the patient. ${ }^{34}$ The DSM-5 criteria was in use in our Cardiac Surgery Department before the start of data collection and the team was familiar with it. Finally, the number of very elderly patients is relatively low in our study, but these numbers will be increasing because of advancement of surgical and anesthetic technique.

Despite these limitations, the strength of this study is based on prospective collection of data and on a very large number of patients included in the analysis. This is a real-life pragmatic evaluation of risk factors in patients undergoing complex cardiac surgery. We included all consecutive patients undergoing cardiac surgery in our department, with very broad inclusion criteria and very few exclusions. This puts our analysis in contrast to other research studies with very selected populations where one must be careful to extrapolate the findings to other patient populations.

Identification of risk factors of post-cardiac surgery delirium defined for a specific patient group allows patients and their caregivers to make informed choices rather than guesses of what can be expected after cardiac surgery. In the population of elderly patients, the risk of postoperative delirium is increased. ${ }^{24}$ Both patients and their families expect health care providers to be able to reliably predict risks associated with cardiac surgery, including neurologic complications, such as postoperative delirium.

\section{Conclusions}

ICU delirium complicates the postoperative course of a significant number of elderly patients undergoing cardiac surgery. Patients above the age of 65 years who experience postoperative delirium have poorer outcome and are more likely to have a prolonged hospitalization, prolonged ICU stay, and longer intubation times, but their 30-day mortality is not increased. In our study, the eight independent risk factors for development of post-cardiac surgery delirium in elderly patients were age, low ejection fraction, diabetes, extracardiac arteriopathy, postoperative atrial fibrillation, pneumonia, elevated creatinine, and prolonged hospitalization time.

\section{Acknowledgments}

We would like to express our gratitude to the patients who provided the data and the staff of the Department of Cardiac Surgery and the Department of Anesthesiology, Intensive Care and Acute Intoxications at the Pomeranian Medical University in Szczecin, Poland.

\section{Author contributions}

$\mathrm{KK}$ designed the study, collected the data, analyzed the results, and wrote the manuscript. AS performed statistical analysis, analyzed the results and provided critical revision of the manuscript. ML designed the study, collected the data, analyzed the results, and provided critical revision of the manuscript. MS collected the data, analyzed the results, and provided critical revision of the manuscript. MB, IR, and MŻ made substantial contributions to the conception of the work and critically revised the manuscript. All the authors read and approved the final manuscript and agree to be accountable for all aspects of the work.

\section{Disclosure}

The authors report no conflicts of interest in this work.

\section{References}

1. Robinson TN, Eiseman B. Postoperative delirium in the elderly: diagnosis and management. Clin Interv Aging. 2008;3(2):351-355.

2. Sockalingam S, Parekh N, Bogoch II, et al. Delirium in the postoperative cardiac patient: a review. J Card Surg. 2005;20:560-567.

3. McPherson JA, Wagner CE, Boehm LM, et al. Delirium in the cardiovascular ICU. Crit Care Med. 2013;41:405-413.

4. Rabiner CJ, Willner AE, Fishman J. Psychiatric complications following coronary bypass surgery. J Nerv Ment Dis. 1975;160:342-348.

5. Sveinsson IS. Postoperative psychosis after heart surgery. J Thorac Cardiovasc Surg. 1975;70:717-726.

6. American Psychiatric Association. Diagnostic and Statistical Manual of Mental Disorders: DSM-5. 5th ed. Arlington, VA: American Psychiatric Association; 2013.

7. Gottesman R, Grega M, Bailey M, et al. Delirium after coronary artery bypass graft surgery and late mortality. Ann Neurol. 2010;67: 338-344.

8. Rudolph J, Inouye S, Jones R, et al. Delirium: an independent predictor of functional decline after cardiac surgery. J Am Geriatr Soc. 2010; 58:643-649.

9. Burkhart C, Dell-Kuster S, Gamberini M, et al. Modifiable and nonmodifiable risk factors for postoperative delirium after cardiac surgery with cardiopulmonary bypass. J Cardiothorac Vasc Anesth . 2010;24:555-559.

10. Tan M, Felde A, Kuskowski M, et al. Icidence and predictors of postcardiotomy delirium. Am J Geriatr Psychiatry. 2008;16:575-583. 
11. Salluh JI, Wang H, Schneider EB, et al. Outcome of delirium in critically ill patients: systematic review and meta-analysis. $B M J$. 2015;350:h2538.

12. Klein Klouwenberg PMC, Zaal IJ, Spitoni C, et al. The attributable mortality of delirium in critically ill patients: prospective cohort study. BMJ. 2014;349:g6652.

13. Ogawa M, Izawa KP, Satomi-Kobayashi S, et al. Preoperative exercise capacity is associated with the prevalence of postoperative delirium in elective cardiac surgery. Aging Clin Exp Res. 2018;30(1):27-34.

14. Ogawa M, Izawa KP, Kitamura A, et al. Preoperative physical activity in relation to postoperative delirium in elective cardiac surgery patients. Int J Cardiol. 2015;201:154-156.

15. Ogawa M, Izawa KP, Satomi-Kobayashi S. Impact of delirium on postoperative frailty and long term cardiovascular events after cardiac surgery. PLoS One. 2017;12(12):e0190359.

16. Van Harten AE, Scheeren TW, Absalom AR. A review of postoperative cognitive dysfunction and neuroinflammation associated with cardiac surgery and anaesthesia. Anaesthesia. 2012;67:280-293.

17. Rudolph J, Ramlawi B, Kuchel G, et al. Chemokines are associated with delirium after cardiac surgery. The journals of gerontology. J Gerontol A Biol Sci Med Sci. 2008;63:184-189.

18. Inouye SK. Prevention of delirium in hospitalized older patients: risk factors and targeted intervention strategies. Ann Med. 2000;32: 257-263.

19. Gosselt AN, Slooter AJ, Boere PR, Zaal IJ. Risk factors for delirium after on-pump cardiac surgery: a systematic review. Crit Care. 2015; 19:346.

20. Lin Y, Chen J, Wang Z. Meta-analysis of factors which influence delirium following cardiac surgery. J Card Surg. 2012;27:481-492.

21. Inouye SK, Leo-Summers L, Zhang Y, Bogardus ST Jr, Leslie DL, Agostini JV. A chart-based method for identification of delirium: validation compared with interviewer ratings using the confusion assessment method. J Am Geriatr Soc. 2005;53:312-318.

22. Choi YH, Kim DH, Kim TY, Lim TW, Kim SW, Yoo JH. Early postoperative delirium after hemiarthroplasty in elderly patients aged over 70 years withdisplaced femoral neck fracture. Clin Interv Aging. 2017;12:1835-1842.

23. Koster S, Hensens AG, van der Palen J. The long-term cognitive and functional outcomes of postoperative delirium after cardiac surgery. Ann Thorac Surg. 2009;87:1469-1474.
24. Norkienė I, Ringaitienė D, Kuzminskaitė V, Šipylaitė J. Incidence and risk factors of early delirium after cardiac surgery. Biomed Res Int. 2013;2013:323491.

25. Kazmierski J, Kowman M, Banach M, et al. Incidence and predictors of delirium after cardiac surgery: results from the IPDACS study. J Psychosom Res. 2010;69:179-185.

26. Hudetz JA, Iqbal Z, Gandhi SD, Patterson KM, Byrne AJ, Pagel PS. Postoperative delirium and short-term cognitive dysfunction occur more frequently in patients undergoing valve surgery with or without coronary artery bypass graft surgery compared with coronary artery bypass graft surgery alone: results of a pilot study. J Cardiothorac Vasc Anesth. 2011;25:811-816.

27. Cerejeira J, Firmino H, Vaz-Serra A, Mukaetova-Ladinska EB. The neuroinflammatory hypothesis of delirium. Acta Neuropathol. 2010; 119:737-754

28. O'Neal JB, Shaw AD. Predicting, preventing, and identifying delirium after cardiac surgery. Perioper Med (Lond). 2016;26:5-7.

29. Rudolph JL, Babikian VL, Birjiniuk V, et al. Atherosclerosis is associated with delirium after coronary artery bypass graft surgery. $\mathrm{J} \mathrm{Am}$ Geriatr Soc. 2005;53:462-466.

30. Lei L, Katznelson R, Fedorko L, et al. Cerebral oximetry and postoperative delirium after cardiac surgery: a randomised, controlled trial. Anaesthesia. 2017;72:1456-1466.

31. Rudolph J, Jones R, Levkoff S, et al. Derivation and validation of a preoperative prediction rule for delirium after cardiac surgery. Circulation. 2009;119:229-236.

32. Clarke SP, McRae ME, Del Signore S, Styra R. Delirium in older cardiac surgery patients: directions for practice. J Gerontol Nurs. 2010; $36: 34-45$

33. Barr J, Fraser GL, Puntillo K, et al; American College of Critical Care Medicine. Clinical practice guidelines for the management of pain, agitation, and delirium in adult patients in the intensive care unit. Crit Care Med. 2013;41:263-306.

34. Inouye SK, Foreman MD, Mion LC, Katz KH, Cooney LM Jr. Nurses' recognition of delirium and its symptoms: comparison of nurse and researcher ratings. Arch Intern Med. 2001;161:2467-2473.
Clinical Interventions in Aging

\section{Publish your work in this journal}

Clinical Interventions in Aging is an international, peer-reviewed journal focusing on evidence-based reports on the value or lack thereof of treatments intended to prevent or delay the onset of maladaptive correlates of aging in human beings. This journal is indexed on PubMed Central, MedLine,

\section{Dovepress}

CAS, Scopus and the Elsevier Bibliographic databases. The manuscript management system is completely online and includes a very quick and fair peer-review system, which is all easy to use. Visit http://www.dovepress. com/testimonials.php to read real quotes from published authors. 